Check for updates

Cite this: RSC Adv., 2019, 9, 2892

\title{
Bi-functional titanium-polydopamine-zinc coatings for infection inhibition and enhanced osseointegration
}

\author{
Lei Wang, $\dagger^{\mathrm{a}}$ Xifu Shang, $\dagger^{\mathrm{b}}$ Yuefeng Hao, $\dagger^{\mathrm{c}}$ Guoyang Wan, ${ }^{\mathrm{c}}$ Lijun Dong, ${ }^{\mathrm{a}}$ \\ Degang Huang, ${ }^{a}$ Xin Yang, (D) Junying Sun, ${ }^{d}$ Qiang Wang, ${ }^{\text {*a Guochun Zha*e }}$ \\ and Xing Yang ${ }^{\star c}$
}

\begin{abstract}
The ideal orthopedic implant coating is expected to both inhibit microbial infection and promote osseointegration. In this study, $\mathrm{Zn}$ ions were immobilized on a Ti substrate via a polydopamine (PDA) chemical surface modification to prepare Ti-PDA-Zn coatings. Scanning electron microscopy (SEM), atomic force microscopy (AFM), energy-dispersive $X$-ray spectroscope (EDS), $X$-ray photoelectron spectroscopy (XPS), contact analysis system, and inductively coupled plasma atomic emission spectrometry (ICP-AES) were used to analyze the morphology, composition, wettability, and zinc ions release of the coatings. The Ti-PDA-Zn coatings demonstrated excellent antibacterial activities in vitro against both Staphylococcus aureus and Escherichia coli. The coatings additionally displayed good biocompatibility, as confirmed by cytoskeletal observations and cell viability assays. Furthermore, the in vivo results confirmed the excellent antibacterial properties and improved osseointegration capability of the Ti-PDA-Zn coating in the presence of $S$. aureus. The present findings indicate that the Ti-PDA-Zn coatings prepared herein have potential application in orthopedic implantation.
\end{abstract}

Received 3rd November 2018
Accepted 9th January 2019

DOI: 10.1039/c8ra09112a

rsc.li/rsc-advances addition, such infection and inflammation reactions can compromise bone integration and cause implant loosening., As a result, complex revision surgeries and prolonged hospitalization, with generally high associated medical costs, are often inevitable., ${ }^{\mathbf{9 1 0}}$ Therefore, the engineering of implant surfaces with reduced infection risks and improved osseointegration capabilities is highly desirable for the clinical success of established titanium implants (Scheme 1).

Recently, zinc has attracted growing attention for various biomedical applications owing to its excellent antibacterial properties and biological activity. It has been proven that zinc ions can interfere with multiple bacteria-related activities, including transmembrane proton translocation, glycolysis, and acid tolerance. ${ }^{11} \mathrm{Hu}$ et al. have reported that $\mathrm{Zn}$-implanted $\mathrm{TiO}_{2}$ coatings exhibited good antibacterial effects against both Escherichia coli (E. coli) and Staphylococcus aureus (S. aureus) as well as excellent biocompatibility. ${ }^{12}$ Wang et al. reported the fabrication of ZnO-modified titanium surfaces that not only exhibited broad-spectrum antibacterial properties, but also could suppress the formation of a bacterial biofilm. ${ }^{13}$ Furthermore, extensive research has confirmed the excellent biological activity of zinc. For instance, Liang et al. demonstrated that Znmodified Ti substrates could improve osteoblast biocompatibility by inhibiting MG-63 apoptosis and otherwise promoting its proliferation. ${ }^{\mathbf{1 4}}$ Yusa et al. developed a zinc-modified titanium surface that could up-regulate expression of osteoblastrelated genes. ${ }^{15}$ Shen et al. demonstrated that $\mathrm{Zn}$-incorporated 

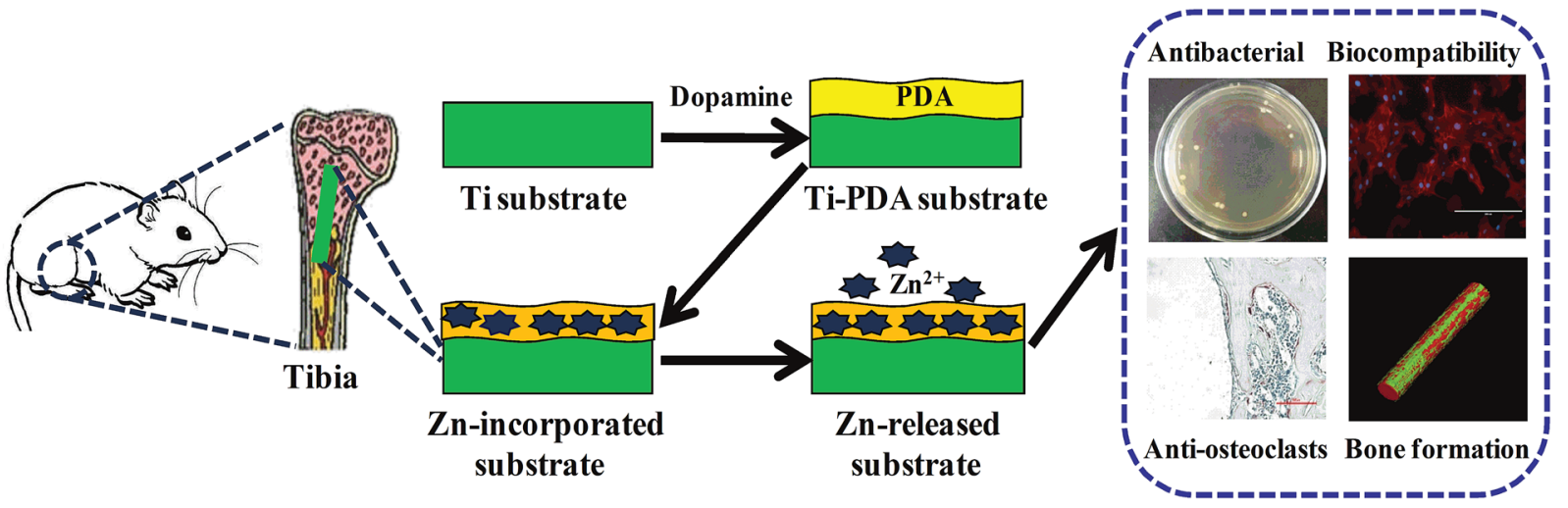

Scheme 1 Schematic diagram of the study.

microrough titanium coatings could enhance bone formation by regulating the proliferation or differentiation of both osteoblasts and osteoclasts. ${ }^{16}$ These studies show that the incorporation of zinc into implants is an ideal approach to endow the implants with both antimicrobial and osteogenic activities.

To date, several methodologies to incorporate zinc into various types of biomaterials have been reported, including plasma spraying, ${ }^{17}$ magnetron sputtering, ${ }^{18}$ plasma immersion ion, ${ }^{19}$ plasma electrolytic oxidation, ${ }^{20}$ and sol-gel ${ }^{21}$ methods. However, most of these approaches require special equipment and sophisticated technologies. Alternatively, inspired by the adhesive proteins, Mfp-5, secreted by mussels in nature, polydopamine (PDA) coatings are generating much interest for use in the surface modification of metallic implants. ${ }^{22,23}$ Under weak alkaline conditions, the polymerization of dopamine is achieved by oxidation of the dopamine to dopamine quinone and formation of 5,6-dihydroxyindole through intermolecular cyclization, the material formed can be deposited as a thin polymerized coating layer on diverse types of material surfaces via covalent and/or non-covalent bonding. ${ }^{24}$ Previous studies have demonstrated the use of polydopamine in successfully depositing active metal ions (e.g., $\mathrm{Ag}, \mathrm{Cu}, \mathrm{Zn}$ ) on various materials. $^{25-27}$ Moreover, numerous studies have confirmed that polydopamine coatings can improve the hydrophilicity and biocompatibility of a biomaterial..$^{28-30}$

In the present study, polydopamine-functionalized titanium substrates were fabricated and subsequently treated with $\mathrm{ZnCl}_{2}$ solution to achieve dual-functionality on the $\mathrm{Ti}$ implants. Animal models were used to test the antimicrobial effect and the osteogenic potential of the Ti-PDA-Zn coatings in the presence of bacteria. In vitro studies were performed to assess whether the Ti-PDA-Zn coatings could endow the Ti implants with both good self-antibacterial performance and biocompatibility. In the in vivo studies, the Ti-PDA-Zn coatings were implanted in a rat model to examine the antibacterial and osseointegration performance of the coatings. As the relationship between $S$. aureus and osteoclasts is rarely examined, ${ }^{31}$ tartrate-resistant acid phosphatase (TRAP) staining and diaminobenzidine (DAB) staining of murine monoclonal antibodies were used to assess the influence of $S$. aureus on osteoclast activity. The findings showed that the Ti-PDA-Zn coatings exhibited both excellent antimicrobial and osseointegration properties. To our knowledge, this is the first study reporting the preparation of zinc-coated PDA-modified titanium surfaces with such dual functionalities.

\section{Materials and methods}

\section{Preparation of Ti-PDA}

Pure Ti plates with dimensions of $5.8 \mathrm{~mm} \times 5.8 \mathrm{~mm} \times 1 \mathrm{~mm}$ and $13 \mathrm{~mm} \times 13 \mathrm{~mm} \times 1 \mathrm{~mm}$ (length $\times$ width $\times$ thickness) were used for the in vitro studies. For the in vivo experiments, pure Ti rods with dimensions of $10 \mathrm{~mm} \times 1.2 \mathrm{~mm}$ (length $\times$ diameter) were used. These materials were supplied by Tianjin Zhengtian Medical Device Company (Tianjin, China). Prior to PDA coating, the Ti plates and rods were washed sequentially with acetone, ethanol, and deionized water under ultrasonication. Dopamine solution $\left(2 \mathrm{mg} \mathrm{mL} L^{-1}\right)$ was prepared by dissolving dopamine hydrochloride (400 mg; Sigma, MO, USA) in Tris-HCl buffer $(200 \mathrm{~mL} ; 10 \mathrm{mM}, \mathrm{pH}=8.5)$. The titanium plates and rods were then soaked in the dopamine solution for $24 \mathrm{~h}$ for PDA coating. The PDA-decorated titanium substrates (Ti-PDA) were ultrasonically cleaned using deionized water and dried overnight in a vacuum oven at room temperature.

\section{Fabrication of Ti-PDA-Zn}

Zinc ions were immobilized on the PDA coatings via soaking. A solution of $\mathrm{ZnCl}_{2}$ (Acros Reagent Co. Ltd., Geel, Belgium) in deionized water was first prepared to achieve a $\mathrm{Zn}$ concentration of $0.2 \mathrm{~mol} \mathrm{~L}^{-1}$. The Ti-PDA substrates were then immersed in the solution for $30 \mathrm{~min}$ to anchor $\mathrm{Zn}$ ions onto the surfaces; the resulting substrates are referred to as Ti-PDA-Zn. Finally, the coatings were ultrasonically cleaned using deionized water, airdried, and sterilized by irradiation prior to subsequent experiments.

\section{Characterization}

The surface morphologies of the pure Ti, Ti-PDA, and Ti-PDA-Zn substrates were observed using scanning electron microscopy (SEM; S-3400, Hitachi, Tokyo, Japan) and atomic force microscopy (AFM; XE-100, Park Systems, USA). The elemental 
composition of the samples was determined by energydispersive X-ray spectroscopy (EDS, QX200, Bruker, Germany) and X-ray photoelectron spectroscopy (XPS; PHI 5802, Physical Electronics, London, UK). The Zn, N, C, O, and Ti profiles were acquired by XPS in conjunction with Ar ion bombardment at a sputtering rate of $\sim 4 \mathrm{~nm} \min ^{-1}$. The surface wettability of the substrates was assessed using a contact analysis system (DSA 25S, Kruss GmbH, Hamburg, Germany); three replicates were measured per sample. All measurements were conducted at room temperature.

\section{Zinc ion release}

The Ti-PDA-Zn samples were immersed in phosphate-buffered saline solution (10 mL; PBS; Hyclone, UT, USA) and incubated at $37^{\circ} \mathrm{C}$ in the absence of stirring for $1,4,7$, and 14 days. These time points were selected according to the biocompatibility tests. The amount of zinc ions released at different intervals of time was measured by inductively coupled plasma atomic emission spectrometry (ICP-AES, Leeman, Ohio, USA). Three samples were used per measurement.

\section{Cell culture}

MC3T3-E1 mouse preosteoblasts (CRL-2594, subclone 14, ATCC) were used to evaluate the biocompatibility of the samples. The cells were incubated at $37{ }^{\circ} \mathrm{C}$ in a $5 \% \mathrm{CO}_{2}$ environment in $\alpha$-minimum essential medium with $10 \%$ fetal bovine serum and 1\% streptomycin/penicillin. The cells were subcultured every 3 days, washed twice with PBS and incubated in a $0.5 \mathrm{~g} \mathrm{~L}^{-1}$ trypsin/ $0.2 \mathrm{~g} \mathrm{~L}^{-1}$ ethylenediaminetetraacetic acid (EDTA) solution (Gibco) for $2 \mathrm{~min}$ at $37{ }^{\circ} \mathrm{C}$ to detach cells from the base of the culture flasks. The subcultured cell solution was then centrifuged at $1000 \mathrm{rpm}$ for $3 \mathrm{~min}$ and resuspended in the complete medium for reseeding on the various surfaces. The cells were seeded at a density of $1 \times 10^{4}$ cells per well in a 96well plate and $2 \times 10^{4}$ cells per well in a 24 -well plate.

\section{Cell morphology observations}

The cells were cultured on Ti, Ti-PDA, and Ti-PDA-Zn substrates ( $5.8 \mathrm{~mm}$ in diameter) in 96-well plates. After 1 day of culture, the samples were washed with PBS and then fixed using 2.5\% glutaraldehyde (Sigma, St. Louis, MO, USA) in PBS for $1 \mathrm{~h}$ and then rinsed three times with PBS for $10 \mathrm{~min}$. Then, the cells were dehydrated in a graded series of ethanol $(30 \%, 50 \%, 70 \%$, $90 \%$, and $100 \%$ ) for $30 \mathrm{~min}$ each and left in $100 \%$ ethanol. The samples were dried using a Critical Point Dryer (CPD030, Leica, Wetzlar, Germany) and sputter-coated gold using an Ion Sputter (SC7620, Quorum Technologies, Lewes, UK) prior to SEM examination. Finally, the morphology of the cells on the surface of the disks was observed by SEM (SEM, S-3400, Hitachi, Tokyo, Japan).

\section{Cytoskeletal observations}

After culturing for 1 day, the cells seeded on the test samples (plates of $13 \mathrm{~mm} \times 13 \mathrm{~mm} \times 1 \mathrm{~mm}$ ) at a density of $2 \times 10^{4}$ cells were briefly rinsed in PBS and fixed for $20 \mathrm{~min}$ in $4 \%$ paraformaldehyde at room temperature. After three rinses with PBS, the cells were permeabilized for 25 min in $0.2 \%$ Triton $\mathrm{X}$ 100 (Sigma, Shanghai, China) and blocked in $0.1 \%$ bovine serum albumin (Sigma, Shanghai, China) in PBS at room temperature in the dark. The cells were then stained in the dark using rhodamine-phalloidin and 4',6-diamidino-2phenylindole. Finally, fluorescence images of the cell morphology and cytoskeletal arrangement were obtained on an EVOS fluorescence microscope (AMG, Thornwood, NY, USA).

\section{Cell viability assays}

Cell proliferation was evaluated using cell counting kit-8 assays (CCK-8, Dojindo Laboratories, Tokyo, Japan). Samples with dimensions of $5.8 \mathrm{~mm} \times 5.8 \mathrm{~mm} \times 1 \mathrm{~mm}$ were placed in a 96well plate. The cell concentration was $1 \times 10^{4}$ cells per well. After 1, 4, and 7 days of culture, the cells were washed twice with PBS. The CCK-8 reagent $(100 \mu \mathrm{L})$ was then added to each well following the manufacturer's directions. After $2 \mathrm{~h}$ of incubation under a $5 \% \mathrm{CO}_{2}$ atmosphere, the absorbance of the solution was measured at a wavelength of $450 \mathrm{~nm}$ using a spectrophotometric microplate reader (Bio-Rad 680, CA, USA). The relative growth rate (RGR) was calculated to analyze the cytocompatibility of the substrates using the following formula:

$$
\mathrm{RGR}=\left(\mathrm{OD}_{\text {sample }} / \mathrm{OD}_{\text {blank }}\right) \times 100 \%
$$

where $\mathrm{OD}_{\text {sample }}$ is the optical density of the Ti, Ti-PDA, or TiPDA-Zn samples and $\mathrm{OD}_{\text {blank }}$ is the optical density of the blank control sample ( $\alpha$-minimum essential medium).

\section{Antibacterial testing}

Plate-counting schemes and live/dead staining were used to investigate the antimicrobial activity of the samples. Grampositive S. aureus (ATCC 25923) and Gram-negative E. coli (ATCC 25922) were selected as the pathogenic microbes. The samples were first sterilized in 75\% ethanol for $2 \mathrm{~h}$ and rinsed three times with sterile PBS. Subsequently, a suspension containing the bacteria at a concentration of $10^{6} \mathrm{cfu} \mathrm{mL}^{-1}$ was introduced onto the sample surface to a density of $100 \mu \mathrm{L} \mathrm{cm}^{-2}$. After incubation at $37^{\circ} \mathrm{C}$ for $24 \mathrm{~h}$, the dissociated bacteria were collected and inoculated onto a standard agar culture plate for $24 \mathrm{~h}$. The antibacterial rate was calculated using the following formula: antibacterial rate $(\%)=(\mathrm{CFU}$ of control $-\mathrm{CFU}$ of experimental group)/CFU of control $\times 100 \%$, where a pristine $\mathrm{Ti}$ sample served as the control, and Ti-PDA and Ti-PDA-Zn samples constituted the experimental groups.

The planktonic bacteria cultured on the tested samples were removed and $100 \mu \mathrm{L}$ of the Live/Dead BacLight staining reagent mixture were added. After staining for 15 min in darkness, the adherent bacteria on the samples were observed with fluorescence microscopy (AMG, Thornwood, NY, USA).

\section{Animal studies}

A total of 12 male Sprague Dawley (SD) rats (weighing 300-350 g) were used for the studies. Four SD rats were allocated to each substrate (Ti, Ti-PDA, and Ti-PDA-Zn), correspondingly forming 
three groups. The rats were anaesthetized with $4 \%$ chloral hydrate $(0.9 \mathrm{~mL}$ per $100 \mathrm{~g})$ prior to surgery. The metaphysis of left tibiae was chosen as the surgical site. After the operating field was shaved and disinfected with iodine, a longitudinal skin incision was made at the proximal tibial metaphysis of the tibia. When the left proximal tibial was exposed by skin incision, a hole was drilled through the proximal tibial metaphysis with a circular drill (1.2 mm diameter) to access the medullary cavity. Then, a $10^{4} \mathrm{cfu} \mathrm{mL}^{-1} S$. aureus suspension $(20 \mu \mathrm{L})$ was injected into the medullary cavity with a microsyringe and the sterile Ti rods were inserted. Finally, the fascia and skin were sutured in a single knot technique (4-0 Prolene, Fa. Ethicon, Norderstedt, Germany). After surgery, the rats were housed in ventilated rooms and given access to water and food. This study was performed in strict accordance with the NIH guidelines for the care and use of laboratory animals (NIH Publication no. 8523 Rev. 1985) and was approved by the Institutional Animal Care and Use Committee of Nanjing Medical University (Nanjing, China).

\section{Micro-CT evaluation}

To evaluate new bone formation around the implants, the specimens were scanned using a high-resolution micro-CT scanner (SkyScan1076, Aartselaar, Belgium) at $65 \mathrm{kV}$ using a $1 \mathrm{~mm}$ Al filter with a resolution of $18 \mu \mathrm{m}$. The threedimensional (3D) high-resolution reconstructed images were obtained using the software provided by the manufacturer. A region of interest (ROI) with a radius of $1.4 \mathrm{~mm}$ from the rods was selected for analysis.

\section{Histological analysis and immunohistochemistry}

After 4 weeks and micro-CT scanning, all animals were euthanized by overdose of chloral hydrate. Four left tibiae in each group were removed and fixed in $4 \%$ paraformaldehyde for $48 \mathrm{~h}$. Then, the samples were decalcified using 10\% EDTA solution for 28 days. All Ti rods were then carefully removed to avoid damage of the newly formed bone tissue, then dehydrated in graded ethanol and embedded in paraffin. Transverse sections (5 $\mu \mathrm{m}$ thick) were collected and stained with hematoxylin-eosin (H\&E). Masson's trichrome staining was used to assess the extent of bacterial infection and osseointegration in vivo. The TRAP staining kit (Sigma, St. Louis, MO, USA) was used to assess osteoclast activity. DAB staining of the murine monoclonal antibody (ab37644; Abcam plc, Cambridge, UK) was performed to investigate the presence of $S$. aureus, as previously reported. ${ }^{32}$

\section{Statistical analysis}

All data are provided as the mean \pm standard deviation (S.D.). The statistical analyses were performed by GraphPad Prism 5 . One-way analysis of variance (ANOVA) tests and student $t$-tests were used. A difference between two groups was considered statistically significant if $p<0.05$.

\section{Results}

\section{Material surface characterization}

The surface morphologies of the samples were examined by SEM (Fig. 1). Both the Ti substrate and Ti-PDA coating displayed similar surface morphologies. In contrast, the Ti-PDA-Zn coating was rougher, and uniformly formed $\mathrm{Zn}$ ion particles could be observed on the surface of the coating. These morphological differences were also confirmed by AFM (Fig. 2). The root-mean-square roughness was significantly increased from $16.31 \mathrm{~nm}$ for the pristine Ti to $55.01 \mathrm{~nm}$ after PDA treatment. After further decoration with $\mathrm{Zn}$, the roughness changed slightly to $53.59 \mathrm{~nm}$. The EDS results further confirmed the successful deposition of $\mathrm{Zn}$ ions on the surface of Ti-PDA-Zn, as indicated by the presence of elemental $\mathrm{Zn}$ compared with the Ti and Ti-PDA samples (Fig. 3). In addition, XPS survey spectra of the Ti, Ti-PDA, and Ti-PDA-Zn films were collected as shown in Fig. 4. Ti and $\mathrm{O}$ peaks could be detected in all samples. In addition, $\mathrm{N}$ peaks at $399.9 \mathrm{eV}$ and $\mathrm{Zn}$ peaks at $1021.7 \mathrm{eV}$ were observed in the Ti-PDA-Zn films, indicating the successful preparation of the PDA coating and immobilization of the $\mathrm{Zn}$ ions. The content of $\mathrm{Zn}$ was calculated to be $\sim 6.26 \%$. A C signal was also observed in all the samples, mainly attributed to surface contamination. The surface wettability of the films was determined and the results are shown in Fig. 5. The water contact angles on Ti-PDA and Ti-PDA-Zn were $15.8 \pm 1.9$ and $18.7 \pm 2.1^{\circ}$, respectively, which were significantly lower than that on pure $\mathrm{Ti}\left(57.1 \pm 3.2^{\circ}\right)$, suggesting that the PDA coating greatly improved the surface wettability of the titanium.

\section{Zinc ion release}

To investigate the influence of the biological environment on the Ti-PDA-Zn coating, the amount of released $\mathrm{Zn}$ ions was measured by ICP-AES (Fig. 6). In the first $24 \mathrm{~h}$ of testing, the TiPDA-Zn sample released $0.71 \pm 0.07 \mathrm{ppm}$ of $\mathrm{Zn}$ ions, and within one week, approximately $1.67 \pm 0.04 \mathrm{ppm}$ of zinc ions were released from the Ti-PDA-Zn sample. The relatively fast $\mathrm{Zn}$ ion release observed in the first week of testing subsequently gradually decreased, and the total amount of $\mathrm{Zn}$ ions released reached $1.70 \pm 0.07 \mathrm{ppm}$ by the end of the immersion test.

\section{Cell morphology and cytoskeletal structures}

Fig. 7 shows the morphology of MC3T3-E1 cells cultured on different samples. There seems to be little difference in cell morphology. However, the cell numbers on the surfaces of the Ti-PDA and Ti-PDA-Zn samples were significantly higher than on the surface of Ti, which might be attributed to the enhanced biocompatibility of the PDA coating. The cytoskeletal structure of the MC3T3-E1 cells was observed by fluorescence microscopy (Fig. 8). Compared with the cells cultured in the presence of TiPDA and Ti-PDA-Zn, the cells cultured in the presence of pure Ti were spread relatively poorly with a spindle shape, and seemed to lack microfilaments. Furthermore, MC3T3-E1 cells cultured at a higher density on the surfaces of Ti-PDA and Ti-PDA-Zn films exhibited better cell spreading, as indicated by the irregular morphology with the development of numerous 

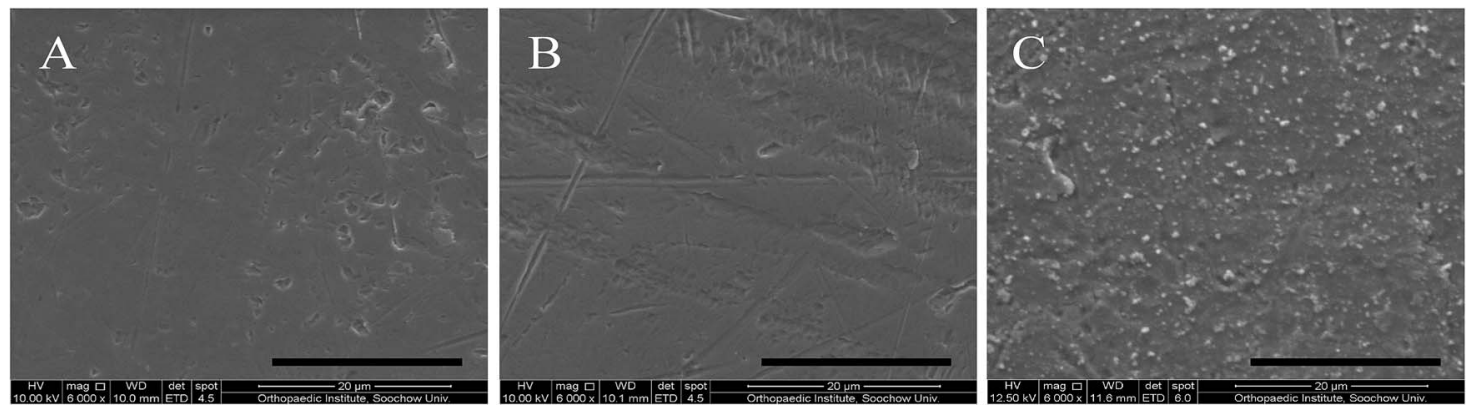

Fig. 1 SEM images of Ti (A); Ti-PDA (B); Ti-PDA-Zn (C). Scale bars, $20 \mu \mathrm{m}$.

lamellipodia and filopodia processes. These results indicated that a PDA coating can promote cellular initial adhesion and spreading.

\section{Cell viability assays}

The effect of the different samples on the proliferative activity of MC3T3-E1 cells was examined by CCK-8 assay. Fig. 9A shows the OD values measured after 1,4 , and 7 days of incubation. After 1 and 4 days of incubation, no differences in the OD values could be observed for all three samples. However, cell proliferation on the Ti sample was superior to that on the Ti-PDA and Ti-PDA-Zn samples $(p<0.05)$ after 7 days of incubation. In addition, no differences could be found in the OD values between the Ti-PDA and Ti-PDA-Zn samples. Fig. 9B shows the calculated RGRs of the MC3T3-E1 cells on the different samples and for different cultivation periods. According to the standard, a biomaterial with a cytotoxicity of grade 0 or grade 1 when RGR is $90-100 \%$ or $75-90 \%$, respectively, implies that this material exhibits no toxicity to the cell. As observed in our studies, the RGR values obtained for the different samples at the different culture times examined were all greater than $75 \%$, indicating that $\mathrm{Ti}$, as well as Ti-PDA and Ti-PDA-Zn showed no cytotoxicity to the MC3T3E1 cells. Thus, Ti-PDA and Ti-PDA-Zn possess good cell biocompatibility similar to Ti.

\section{Antibacterial testing}

Fig. 10 shows representative images of bacteria colonies on $\mathrm{Ti}$, Ti-PDA, and Ti-PDA-Zn after $24 \mathrm{~h}$ of incubation. Numerous bacterial colonies were found on the Ti and Ti-PDA samples, suggesting that both samples did not have any antibacterial properties. In contrast, only several colonies were observed on the Ti-PDA-Zn sample, which demonstrated that Ti-PDA-Zn exhibited a very high antibacterial ability. After exposure to the fluorescent stain, live bacteria with intact membranes appear green while dead bacteria with damaged membranes appear red. As shown in Fig. 11, the vast majority of bacteria on $\mathrm{Ti}$ and Ti-PDA were viable, but the majority of bacteria on TiPDA-Zn were inactivated. The calculated antibacterial rates for Ti-PDA and Ti-PDA-Zn against $S$. aureus and E. coli using the plate-counting method are shown in Fig. 12. The antibacterial rates against $S$. aureus for Ti-PDA and Ti-PDA-Zn were $20.5 \%$ and $94.1 \%$, respectively. For $E$. coli, the same trend was obtained; the antibacterial rates for Ti-PDA and Ti-PDA-Zn were $17.9 \%$ and $89.5 \%$, respectively.

\section{Micro-CT evaluation}

Micro-CT was used to analyze the formation and integration of new bone after implantation for 4 weeks; representative microCT images are depicted in Fig. 13A-C. The Ti and Ti-PDA implants were surrounded by only a few bone trabeculae. In comparison, more bone trabeculae could be observed around the Ti-PDA-Zn implant. These results were confirmed by the BV/ TV data (Fig. 13D). Under the same threshold and volume of interest (VOI) for CT scanning, the Ti-PDA-Zn implant exhibited a higher percentage of BV/TV than the Ti and Ti-PDA implants, indicating that the Ti-PDA-Zn sample possessed enhanced osseointegration performance.

\section{Histological analysis and immunohistochemistry}

The transverse decalcified sections of the rat tibia from the different groups examined are shown in Fig. 14 and 15. H\&E and Masson's trichrome staining were used to observe
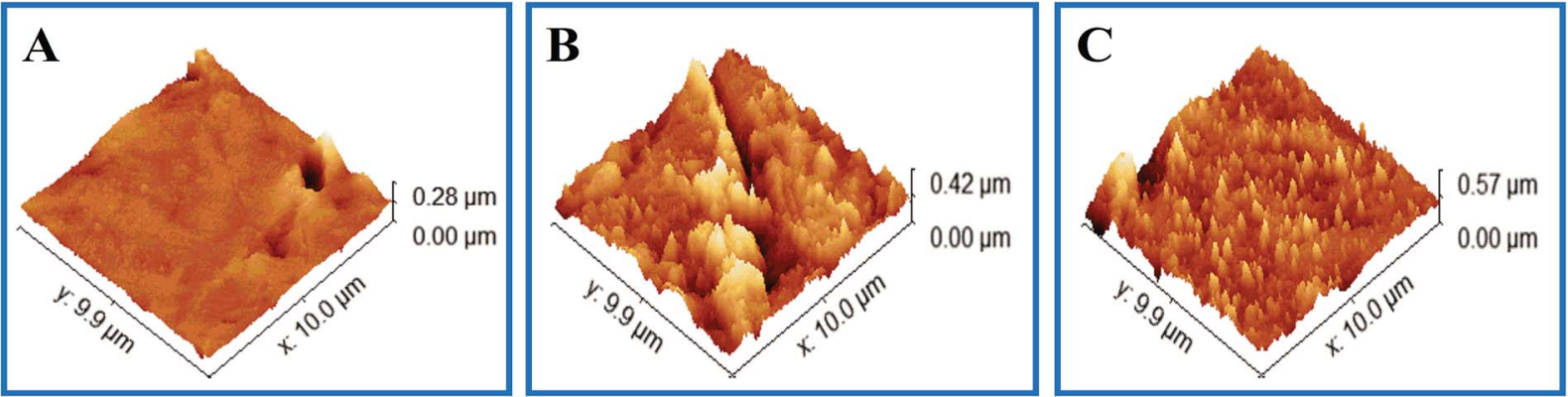

Fig. 2 3D topographical AFM images of Ti (A); Ti-PDA (B); Ti-PDA-Zn (C). 

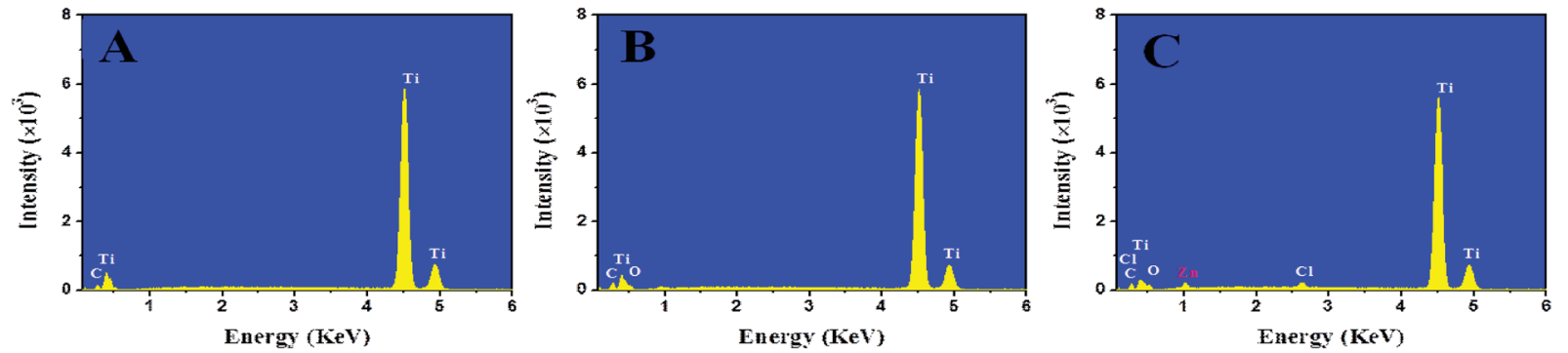

Fig. 3 EDS spectrum of Ti (A); Ti-PDA (B); Ti-PDA-Zn (C).

bacterial infection and osseointegration, while osteoclast activity and bacterial residue were confirmed by TRAP staining and immunohistochemical analysis. Four weeks after surgery, the bone tissues around the Ti and Ti-PDA implants were destroyed by bacterial infection, as confirmed by the large number of neutrophils observed inside the medullary cavity by H\&E staining. The corresponding TRAP staining and immunohistochemical analysis also demonstrated the accumulation of osteoclasts and bacterial residues around the tibia medullary cavity for the Ti and Ti-PDA implants, suggesting the occurrence of bone resorption. In comparison, a large amount of woven bone was detected at the implant-tissue interface for the Ti-PDA-Zn sample as shown by Masson's trichrome staining. Moreover, no detectable signs of bacterium infection were observed for the Ti-PDA-Zn implant, suggesting that the Ti-PDA-Zn coating effectively eradicated bacteria and prevented implant-related infections.

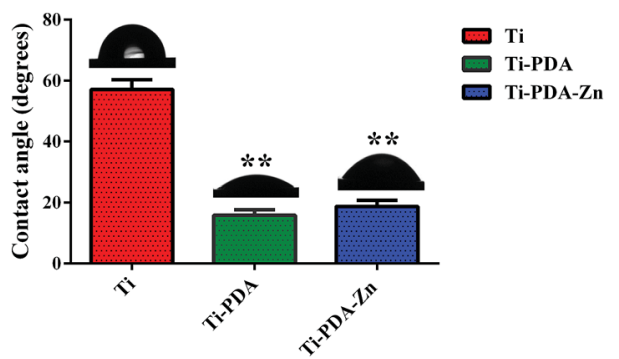

Fig. 5 Images of water drops on the surface of Ti, Ti-PDA, and TiPDA-Zn coatings. ** $p<0.01$ vs. Ti.

\section{Discussion}

Orthopedic implants are widely used and highly successful treatments for musculoskeletal issues including trauma, osteoporotic diseases, bone cancer, and joint and spinal diseases. It has been estimated that by the end of 2030 , the number of total
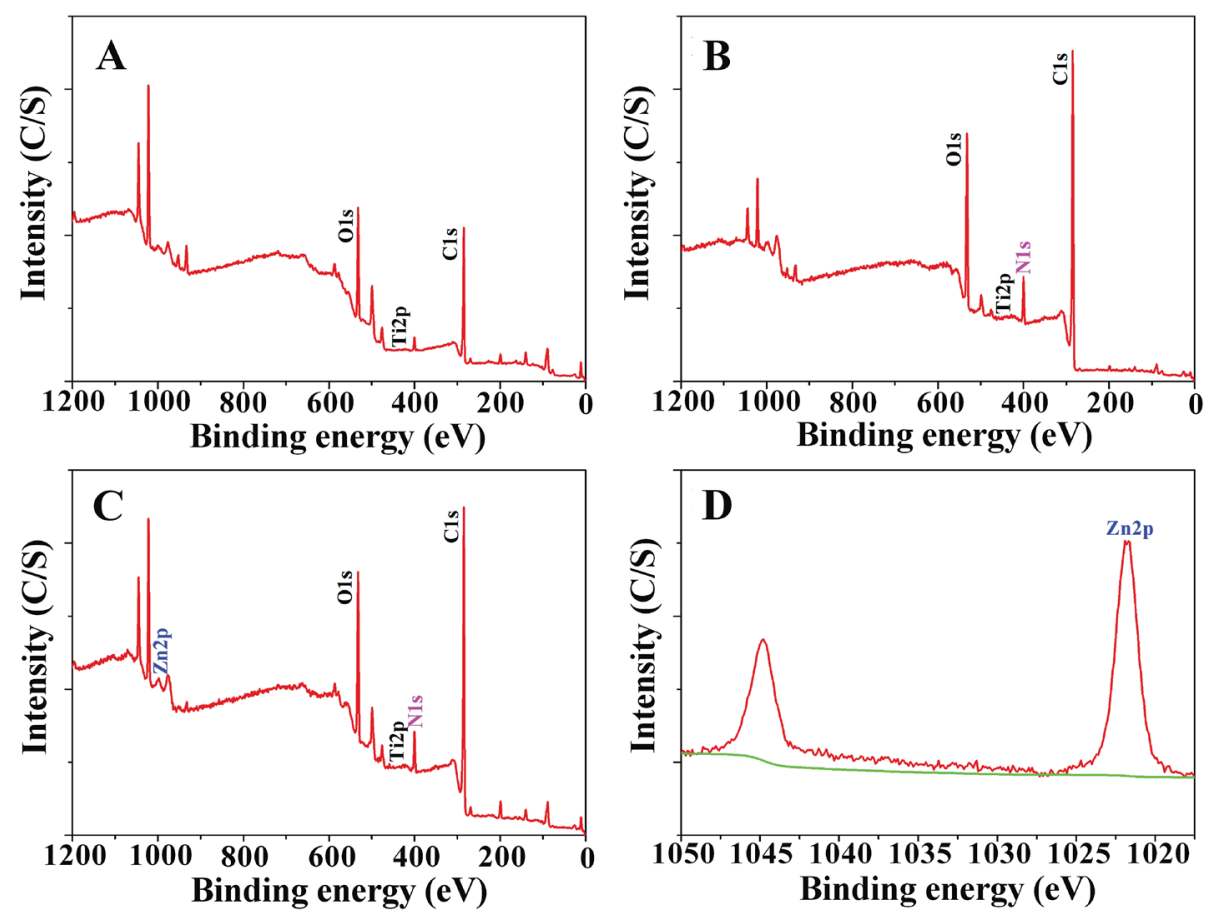

Fig. 4 XPS survey spectra of Ti (A); Ti-PDA (B); Ti-PDA-Zn (C); and high-resolution spectra of Zn 2p (D). 


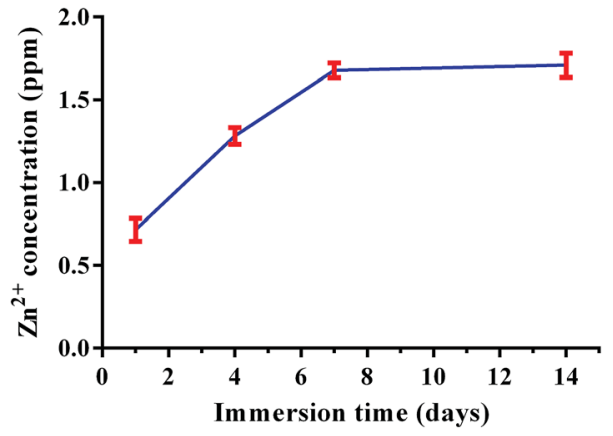

Fig. $6 \mathrm{Zn}^{2+}$ concentrations in PBS solution after immersion for 1, 4, 7, and 14 days.

hip replacements and total knee arthroplasties will rise by $174 \%$ (572 000 procedures) and 673\% (3.48 million procedures), respectively, in the United States. ${ }^{33}$ However, the success of these treatments can be undermined by implant-related infections and insufficient osseointegration, the two leading causes of implant failure and complex revision surgeries. ${ }^{34}$ For total joint arthroplasty, $20 \%$ of implant failures are attributed to infection. ${ }^{35,36}$ In addition, recent studies point to the key role that microbes may play in initiating or accelerating implant failure pathways even in cases where infection is not the primary cause. ${ }^{37}$ The annual cost for treating periprosthetic joint infection is projected to exceed $\$ 1.62$ billion by $2020 .^{38} \mathrm{As}$ demands for joint arthroplasty are expected to increase substantially over the coming decade, improvements in the overall function and lifetime of orthopedic implants are highly desirable not only to improve patients' well-being, but also to alleviate a huge economic burden on society. From the perspective of clinical applications, the ideal orthopedic implant coating to prevent or treat periprosthetic joint infections will likely be multifunctional to meet the various requirements. The implanted biomaterial must possess broadspectrum antibacterial performance as a wide variety of bacteria can infect an implant, including Gram-positive and Gram-negative bacteria. ${ }^{39}$ The implant also needs to exhibit excellent biological properties to promote osseointegration at the bone-implant interface, thereby limiting the chances of infection leading to osteolysis and implant loosening. ${ }^{34,40}$

In the present study, we have successfully fabricated $\mathrm{Zn}$ surface-modified Ti substrates via PDA chemical surface modification to achieve both excellent biological and antibacterial performance. The successful formation of the PDA coating on the titanium substrate and anchoring of the $\mathrm{Zn}$ ions to the PDA coating through coordination interaction were confirmed by EDS and XPS (Fig. 3 and 4). It is known that Grampositive Staphylococcus bacteria, most prominently $S$. aureus and $S$. epidermidis contribute to $70 \%$ of orthopedic implant infections, whereas Gram-negative bacteria, such as E. coli and Klebsiella pneumoniae cause approximately $5 \%$ of infections. ${ }^{41-43}$ In addition, inhibition of invasive-bacteria spread as early as possible, especially within the first $6 \mathrm{~h}$ of the implantation surgery, helps to reduce the risk of biofilm formation, as the invading bacteria have not yet begun rapid proliferation. ${ }^{44,45} \mathrm{We}$ chose representative Gram-positive $S$. aureus and Gram-negative $E$. coli to evaluate the broad-spectrum antibacterial properties of the Ti-PDA-Zn coatings. The results showed that the Ti-PDA-Zn coating could effectively inhibit the growth of both $S$. aureus and E. coli, which may be attributed to the incorporation of $\mathrm{Zn}$ ions on the surface. Previous studies have confirmed that the antibacterial mechanism of zinc ion-decorated biomaterials can be mainly attributed to the destruction of cell membranes and the production of reactive oxygen species (ROS), eventually killing the bacterial cells. ${ }^{16,46} \mathrm{Ning}$ et al. have reported that the minimal inhibition concentration of $\mathrm{Zn}$ ions against both $S$. aureus and E. coli is $10^{-7} \mathrm{~mol} \mathrm{~L}^{-1} \cdot{ }^{46}$ However, the highest concentration of Zn ions measured by ICP-AES in our study was $\sim 1.7 \mathrm{ppm}$ (Fig. 6). Therefore, the most likely antibacterial mechanism of the Ti-PDA-Zn coating is the generation of ROS. In addition, our studies demonstrate that $S$. aureus is more sensitive to the coating than E. coli, which may be attributed to differences in the cell membrane structures of the Gram-positive and Gramnegative bacteria. ${ }^{12,47}$

Assessing the biocompatibility of a coating is as important toward assessing the potential of the coating in biomedical applications. Zinc is an essential trace element that plays an important role in the structure and function of proteins and
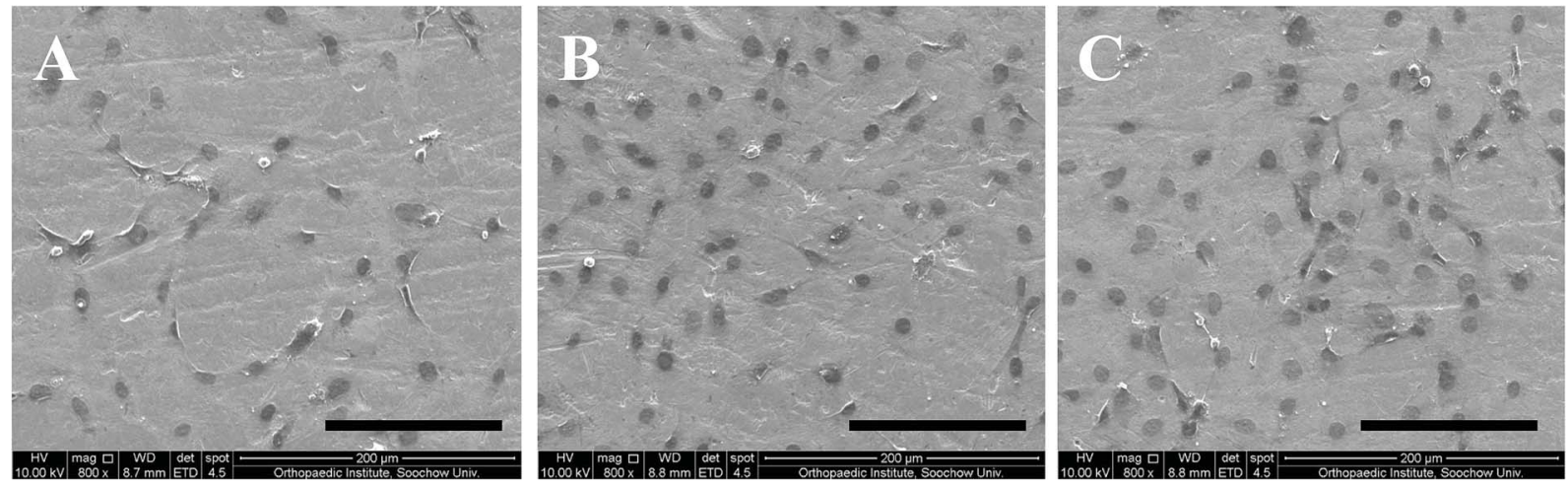

Fig. 7 Morphology of MC3T3-E1 cells cultured on Ti (A), Ti-PDA (B), and Ti-PDA-Zn (C) substrates for 24 h, as observed using SEM. Scale bars, $200 \mu \mathrm{m}$ 
$\mathbf{T i}$
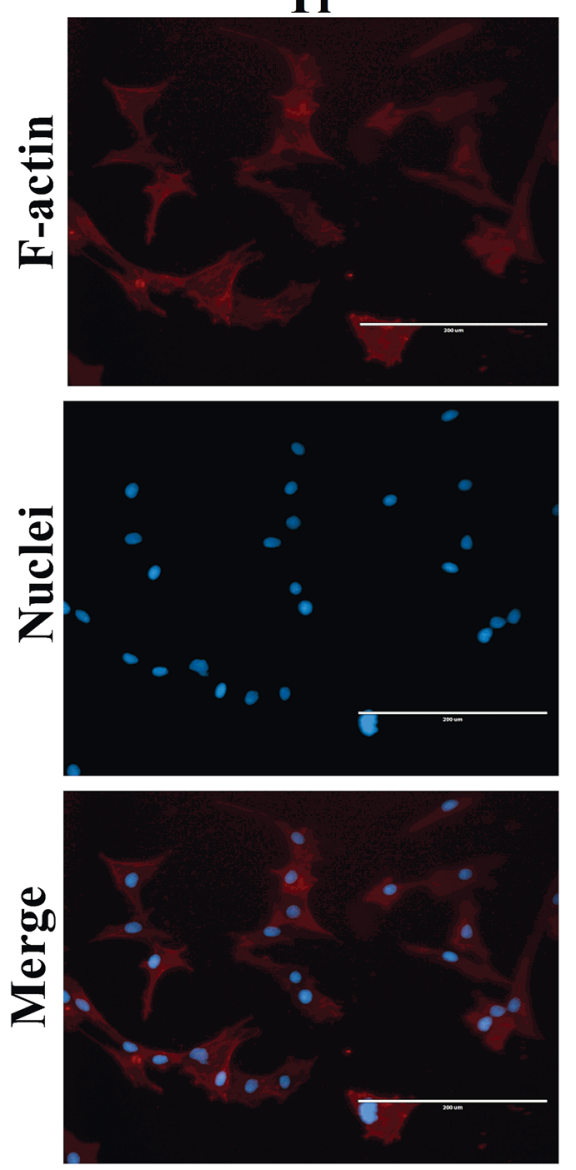

Ti-PDA
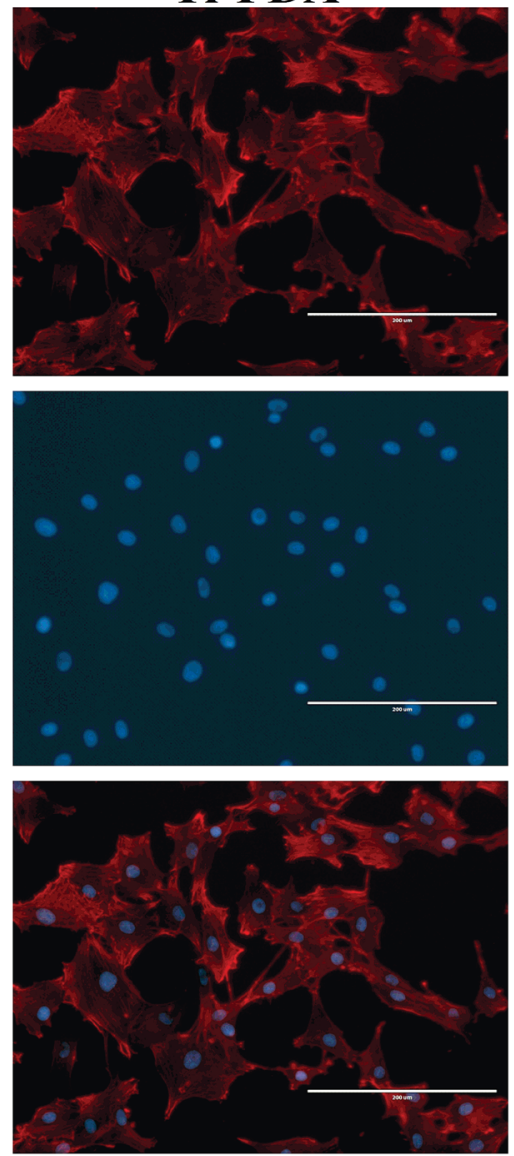

Ti-PDA-Zn
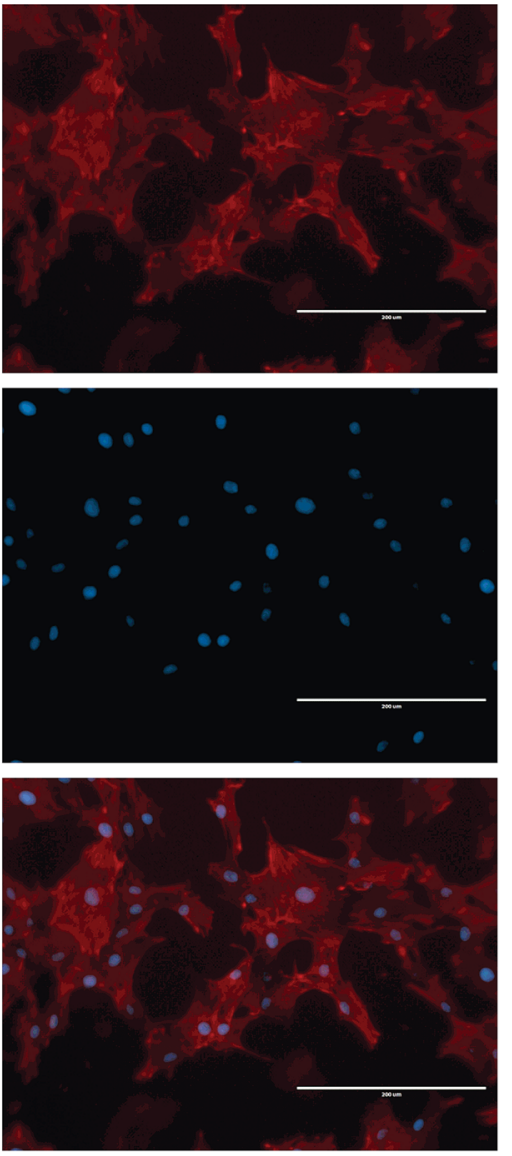

Fig. 8 Fluorescent images of MC3T3-E1 cells cultured on various surfaces for $24 \mathrm{~h}$ with actin stained with phalloidin (red) and nuclei stained with DAPI (blue). Scale bars, $200 \mu \mathrm{m}$.

a variety of cellular processes, including DNA synthesis, enzyme activity, and cell division. ${ }^{48,49}$ In contrast, $\mathrm{Zn}$ ions in high concentrations would induce cellular toxicity. ${ }^{49,50}$ In our study, the in vitro biocompatibility of the Ti-PDA-Zn coating was evaluated using MC3T3-E1 cells. It is well known that the initial adhesion of cells to the surface of an implant is a key step for subsequent cell proliferation and differentiation. The results of SEM and fluorescence microscopy showed that the amounts and skeletal structures of MC3T3-E1 cells on the different titanium substrates were significantly different (Fig. 7 and 8). The amount of MC3T3-E1 cells on the Ti-PDA and Ti-PDA-Zn surfaces was significantly higher than on the Ti surfaces. In addition, compared with the Ti substrate, the MC3T3-E1 cells incubated with the Ti-PDA and Ti-PDA-Zn coatings displayed better cell adhesion and spreading, as indicated by the higher cell density and irregular cell morphology with the development of numerous lamellipodia and filopodia processes. These results indicate that the PDA coating can promote initial cellular adhesion and spreading, which is consistent with the findings from previous studies. ${ }^{28,29}$ According to the concept of the "race for the surface" as laid out by Gristina, if host cells first occupy the surface of the implant, this not only promotes tissue
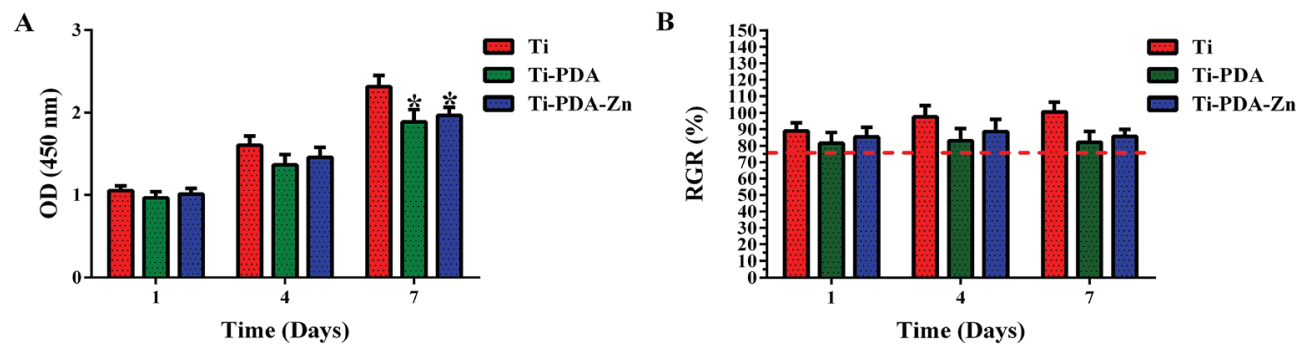

Fig. 9 (A) OD values in CCK-8 tests with Ti, Ti-PDA, and Ti-PDA-Zn coatings; (B) RGR values of cp-Ti, Ti-PDA, and Ti-PDA-Zn coatings with different incubation durations. ${ }^{*} p<0.05$ vs. Ti. 

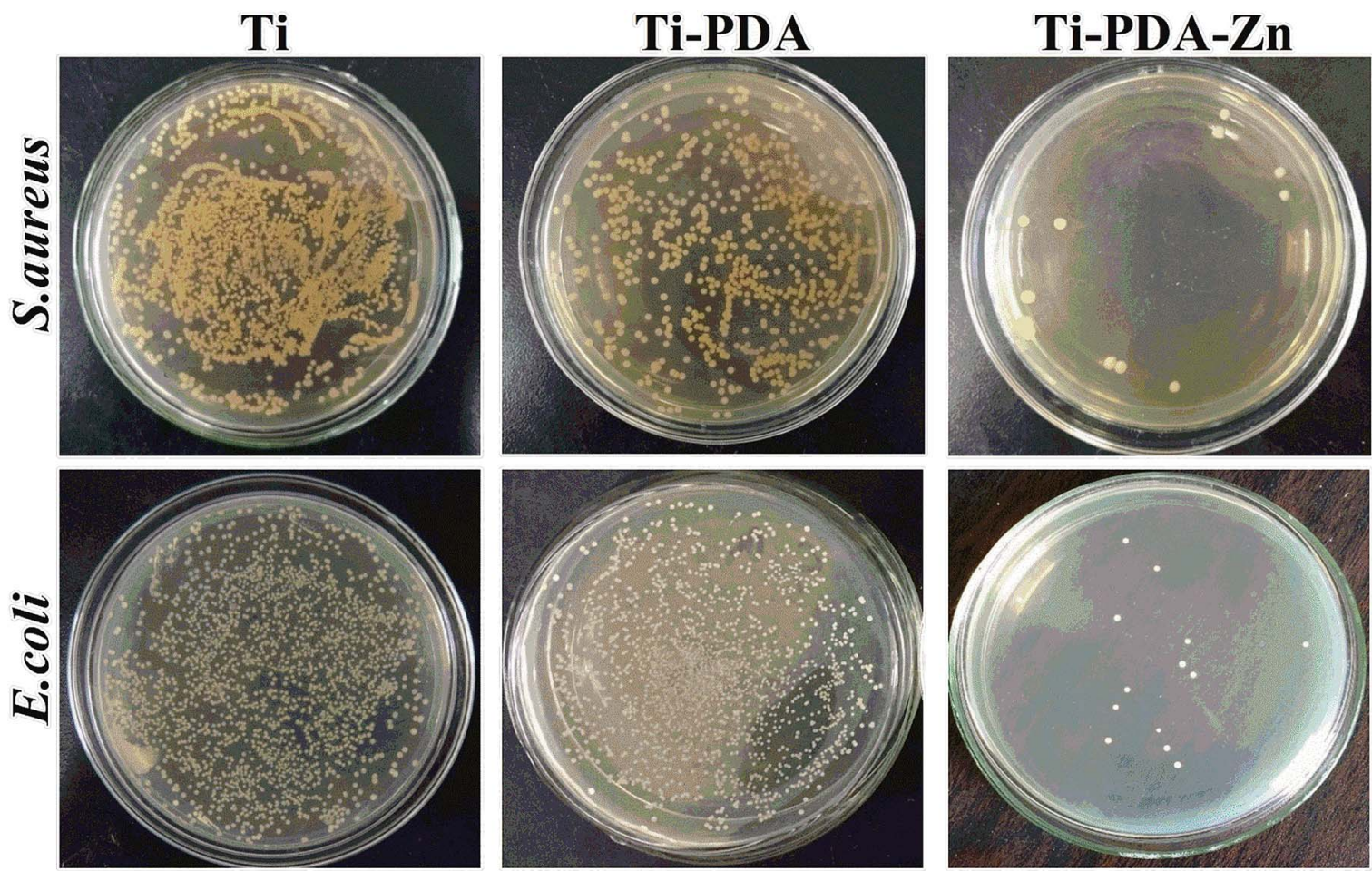

Fig. 10 Representative images of bacteria colonies on Ti, Ti-PDA, and Ti-PDA-Zn substrates after culturing for $24 \mathrm{~h}$.

integration, but also helps build defensive barriers against the attachment and colonization of microbes. ${ }^{51}$ To further investigate the cytotoxicity of the different Ti samples, the proliferative activity of MC3T3-E1 cells was determined by CCK-8 assay and the RGR was calculated. The results showed that the different $\mathrm{Ti}$ samples exerted no significant toxicity on the cells (Fig. 9). After culturing for 1 and 4 days, no differences could be found in the OD values of the Ti, Ti-PDA, and Ti-PDA-Zn samples. However, cell proliferation activity on the Ti-PDA and Ti-PDA-Zn samples was slightly lower than that on the Ti substrate $(p<0.05)$. In addition, the calculated RGR values at different culture times were all higher than $75 \%$, implying that the cell biocompatibility of both the Ti-PDA and Ti-PDA-Zn coatings is as good as that of the Ti substrate. Previous studies have confirmed that $\mathrm{Zn}$ ion concentrations under $2 \mathrm{ppm}$ are safe and would not lead to obvious cytotoxicity. ${ }^{16}$ In our case, the release of $\mathrm{Zn}$ ions was measured by ICP-AES, and the highest concentration of $\mathrm{Zn}$ ions detected was $1.70 \pm 0.07 \mathrm{ppm}$ at 14 days, which is below the $2 \mathrm{ppm}$ safe concentration. The above results thus provide
$\mathbf{T i}$
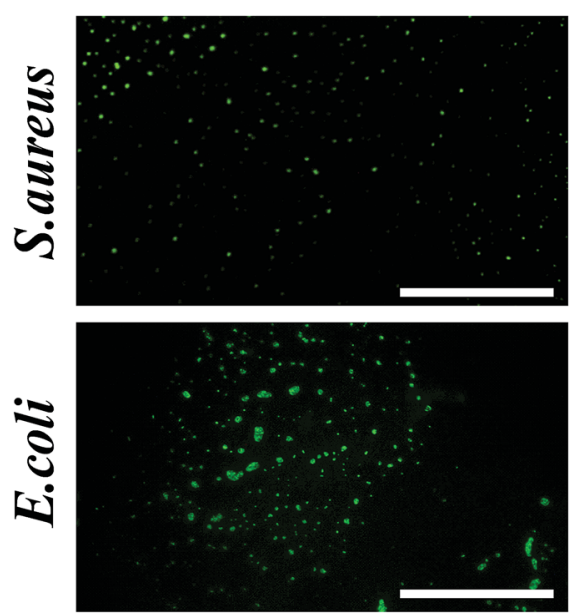

Ti-PDA
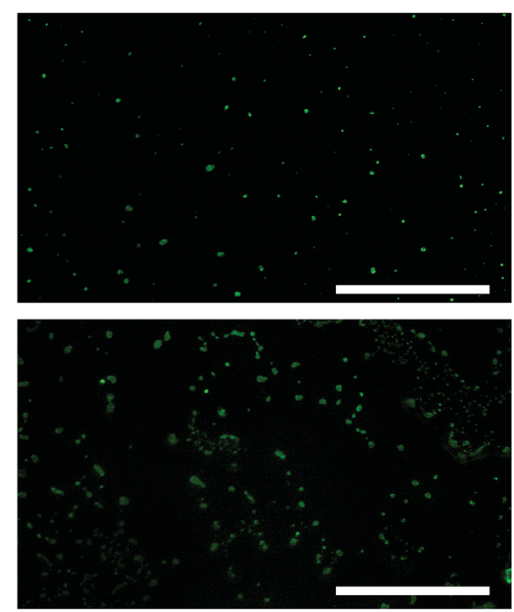

Ti-PDA-Zn
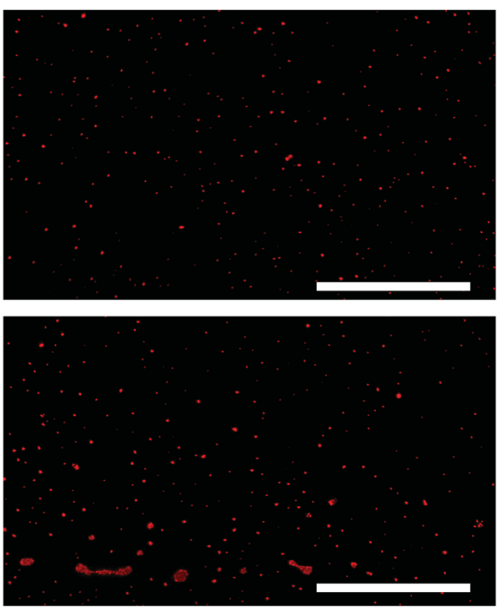

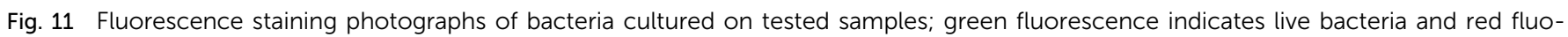
rescence indicates dead bacteria. Scale bars, $20 \mu \mathrm{m}$. 
A

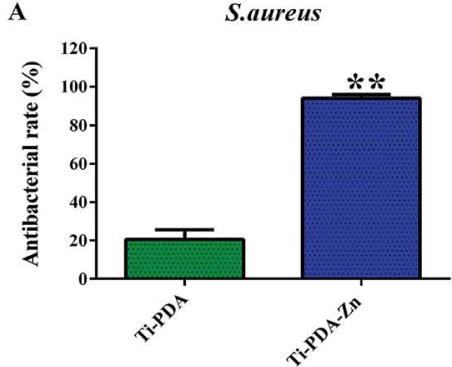

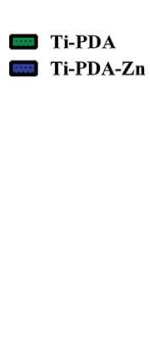

B

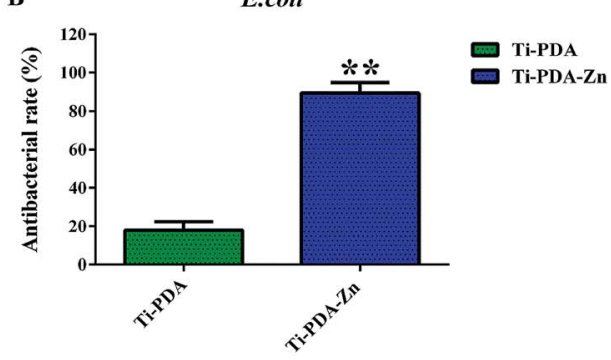

Fig. 12 Calculated antibacterial rates of Ti-PDA and Ti-PDA-Zn substrates against S. aureus (A) and E. coli (B) using the plate-counting method. $* * p<0.01$ vs. Ti-PDA.

a strong basis for supporting the excellent antibacterial activities and good biocompatibility displayed by Ti-PDA-Zn coatings.

In 1975, Schurman et al. proposed the first model of joint infection in rabbits. ${ }^{52}$ Since then, a variety animal models of periprosthetic joint infection have been successfully established, which not only help to better study the pathogenesis of implant-related infections, but also help to better evaluate the antibacterial properties and osseointegration effects of implanted biomaterials. These animal models can mimic both the micro-environment of the infection progress and bone growth. ${ }^{53,54}$ Imaging and histology analyses are currently the main methods used to assess the osteogenic and anti-infection properties of biomaterials in vivo. ${ }^{55}$ Contrary to the traditional $\mathrm{X}$-ray examination, micro-CT analysis can quantitatively evaluate the infection-induced morphological changes of bone surrounding the implants via 3D reconstruction of the scanning
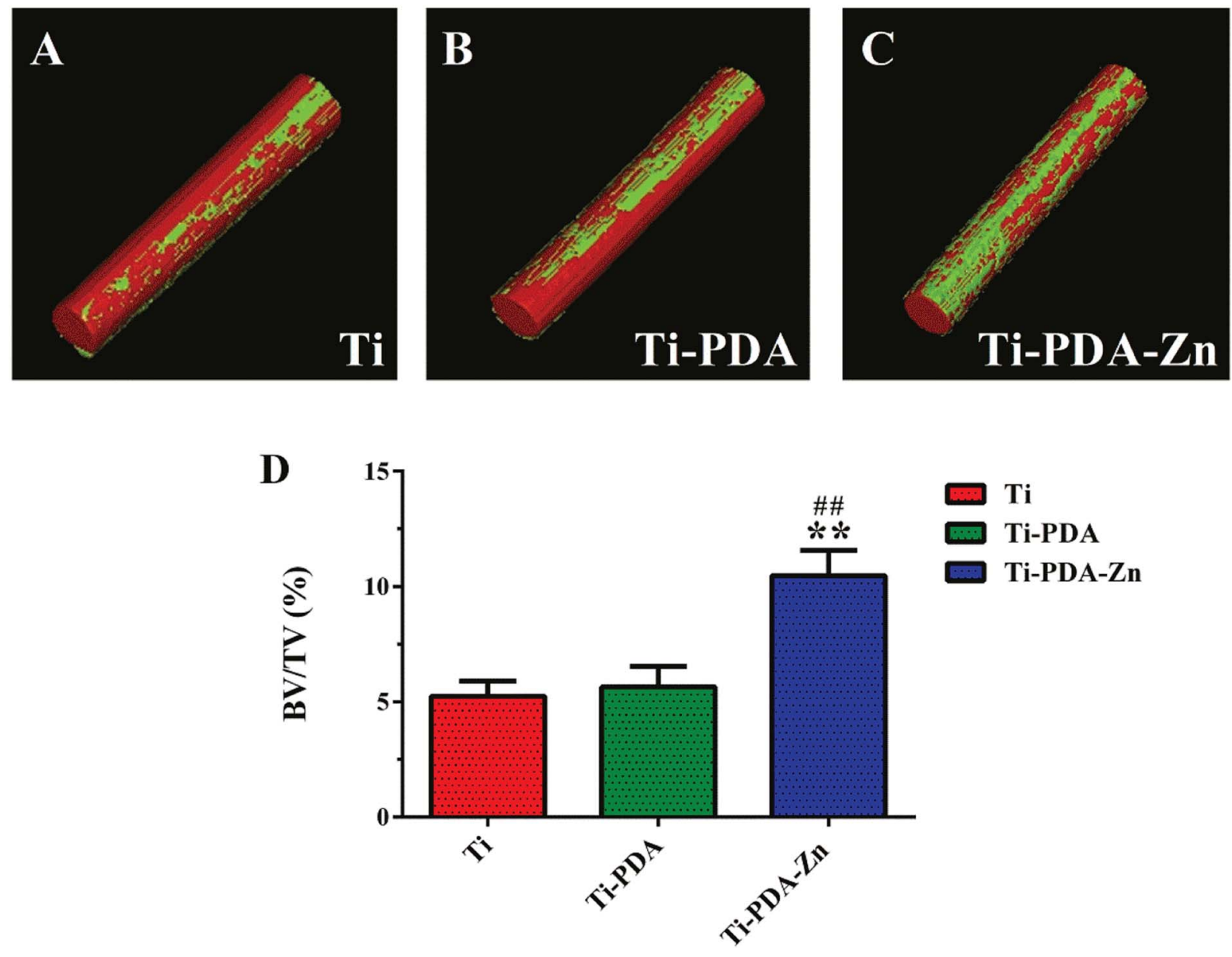

Fig. 13 Micro-CT 3D reconstructed models showing the status of osseointegration around Ti (A), Ti-PDA (B), and Ti-PDA-Zn (C) at 4 weeks after implantation (the red color represents the implant and the green color represents the bone tissue). (D) Bone volume fraction (BV/TV) of the three groups obtained from analysis of the Micro-CT data $(n=4) .{ }^{* *} p<0.01$ vs. Ti and ${ }^{\# \#} p<0.01$ vs. Ti-PDA. 

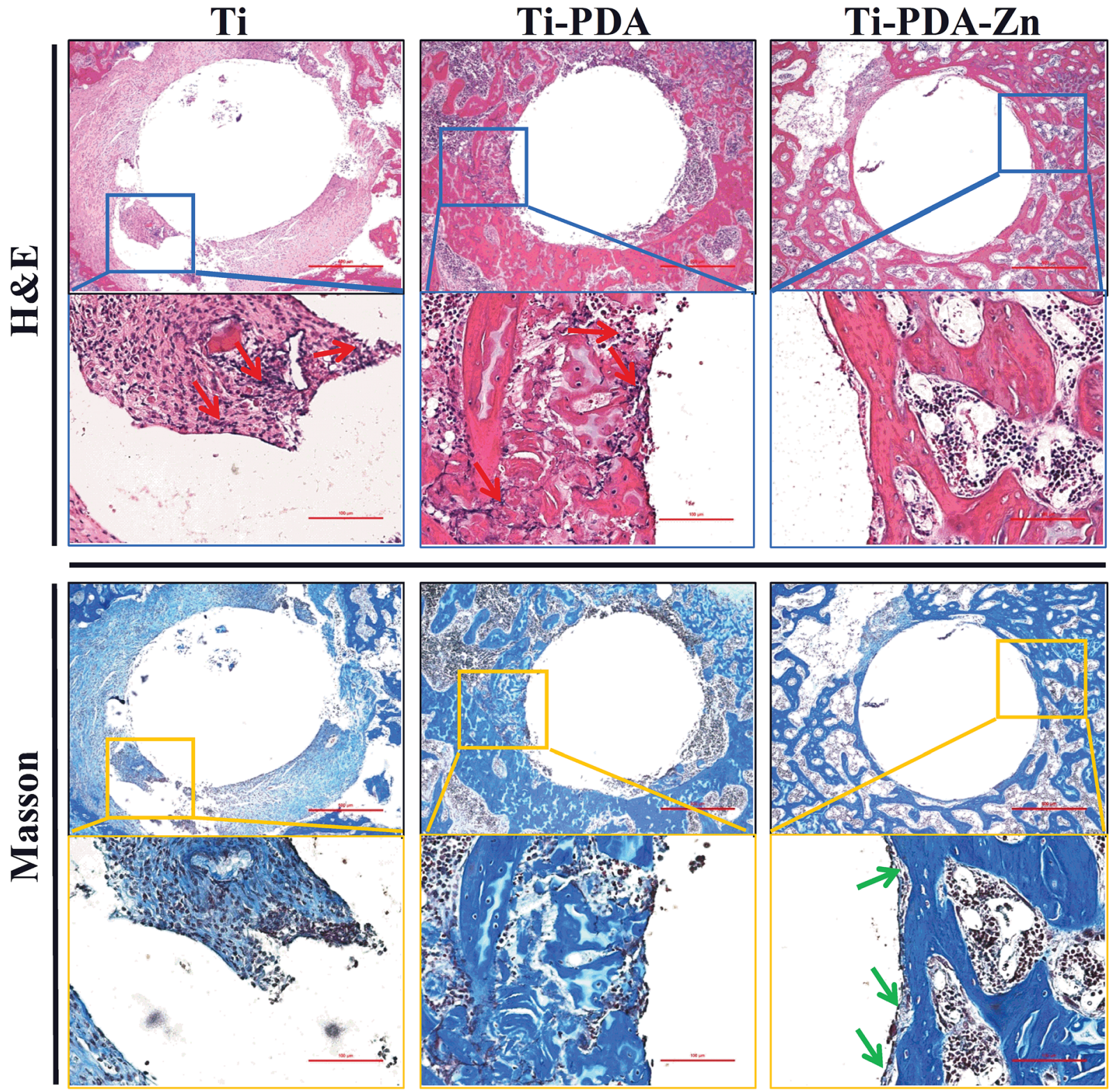

Fig. 14 High magnification histological images of H\&E and Masson' trichrome staining at 4 weeks after surgery. Scale bars, $500 \mu m$.

data and quantitative measurements of the ROI by imaging processing and analysis software. ${ }^{55}$ There are few research studies related to assessing the osteogenic properties of $\mathrm{Zn}-$ decorated biomaterials in the presence of bacteria, although a number of experiments in vitro and in vivo have proved the osteogenic and anti-infection properties of zinc-related biomaterials separately. ${ }^{12,16,48,56,57}$ An intramedullary implant approach was used herein to mimic periprosthetic infection, and the performance of the materials was systematically evaluated using micro-CT and histology analyses and immunohistochemistry. It is well known that stable connections in boneimplant interfaces are crucial to the clinical success of $\mathrm{Ti}$ implants. At the same threshold and VOI, CT scanning indicated that the Ti-PDA-Zn group had a higher percentage of BV/ TV than the Ti and Ti-PDA samples, which agrees with the results of osteogenic differentiation of Zn-incorporated biomaterials. $^{14,15,57}$ Details at the bone-implant interface were then investigated by histological and immunohistochemistry staining. Four weeks after implantation, the bone tissues around the Ti and Ti-PDA implants were destroyed by implant-related osteomyelitis, as confirmed by the large number of neutrophils, and osteoclastic bone resorption, as well as bacterial residues around the tibia medullary cavity. In contrast, no sign of bacterial infection or bone resorption was detected around the Ti-PDA-Zn implant. Moreover, a large amount of woven bone was observed at the implant-tissue interface for the TiPDA-Zn implant as shown by Masson's trichrome staining, which was consistent with the micro-CT results. It is believed that $S$. aureus exerts an effect on both osteoblasts and osteoclasts. Osteoblast infection by $S$. aureus can lead to reduced 

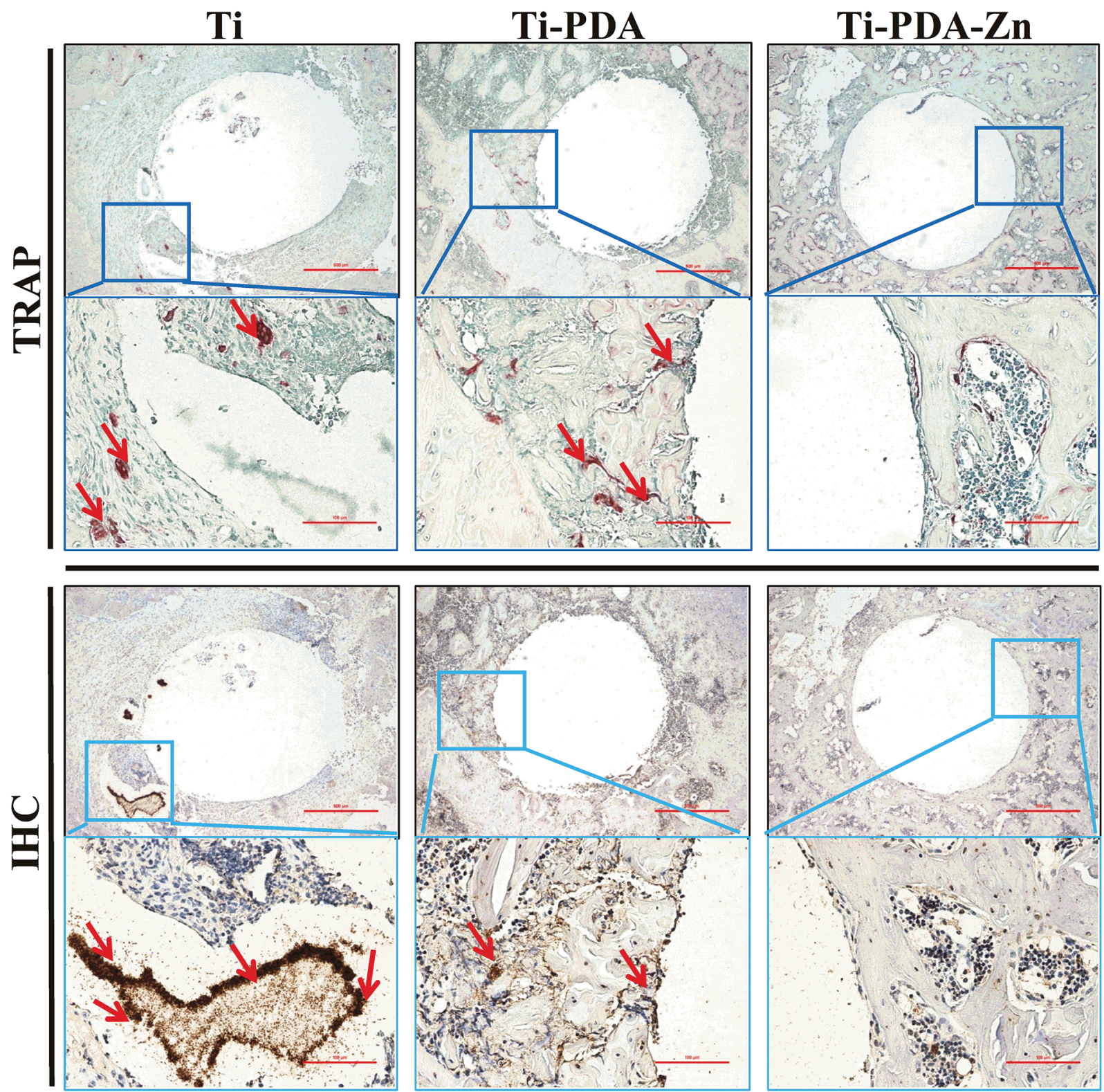

Fig. 15 High magnification histological images of TRAP and immunohistochemical staining at 4 weeks after surgery. Scale bars, $500 \mu m$.

bone formation activity and osteoblast death. ${ }^{58}$ Osteoclasts infected by $S$. aureus can enhance bone resorption activity, and the aggregation of osteoclasts in the infected areas is mediated by the pro-inflammatory and pro-osteoclastogenic factors resulting from immune responses, ${ }^{31}$ which is consistent with the results of histological sections and immunohistochemistry staining in our research. Taken together, the results from the animal model of implant-related infections demonstrated that the Ti-PDA-Zn coating possesses both excellent antibacterial properties and improved osseointegration capability in the presence of $S$. aureus in vivo. These properties are mainly attributable to the antibacterial effects of the zinc ions, which reduce the activity of the osteoclasts mediated by $S$. aureus and reduce infected bone destruction.

The results from the in vitro and in vivo studies demonstrated that the Ti-PDA-Zn coating exhibits excellent antibacterial activity, good biocompatibility, as well as enhanced osseointegration performance. Thus, it is expected that Ti-PDA-Zn can help prevent surgical site infections associated with total joint replacement in the early postoperative period.

\section{Conclusion}

We have developed a bi-functional film in which zinc ions are immobilized on a Ti substrate via PDA chemical surface modification. The resulting Ti-PDA-Zn coating demonstrated excellent antibacterial activities and good biocompatibility in vitro. The results from the animal model of implant-related infections demonstrated that the Ti-PDA-Zn coating displayed both excellent antibacterial properties and enhanced osseointegration capability in the presence of $S$. aureus in vivo. In addition, the preparation of the coating has many advantages, 
including ease-of-use, versatility, and high stability under physiological conditions. Together, the findings from this study indicate that the Ti-PDA-Zn coating is a promising candidate for orthopedic implants, exhibiting both antimicrobial and regenerative properties.

\section{Author contributions}

Conceptualization, Lei Wang, Xifu Shang, and Yuefeng Hao; methodology, Guoyang Wan, Lei Wang, Xifu Shang, and Yuefeng Hao; validation, Lijun Dong and Degang Huang; formal analysis, Junying Sun, and Xing Yang; data curation, Xin Yang; writing-original draft preparation, Lei Wang, Xifu Shang and Yuefeng Hao; writing-review and editing, Qiang Wang, Guochun Zha, and Xing Yang; project administration, Qiang Wang, Guochun Zha, and Xing Yang.

\section{Conflicts of interest}

There are no conflicts to declare.

\section{Acknowledgements}

This work was supported by the Special Scientific Research Fund for Introducing Talents in Yijishan Hospital, National Natural Science Foundation of China (Grant no. 81472060), Program for Construction of Suzhou Clinical Medical Center of Orthopaedics and Burns (Grant no. szzxj201506), Program for Introduction of Clinical Medical Teams to Suzhou (Grant no. szyjtd201714), Program from Jiangsu Health and Health Committee (Grant no. H2018027) and Social Development Program from Suzhou Science and Technology Bureau (Grant no. sys201427, sysd2015118, sysd2017170, sysd2018207).

\section{References}

1 Y. Kirmanidou, M. Sidira, M. E. Drosou, V. Bennani, A. Bakopoulou, A. Tsouknidas, N. Michailidis and K. Michalakis, BioMed Res. Int., 2016, 2016, 2908570.

2 W. J. Metsemakers, K. Kortram, M. Morgenstern, T. F. Moriarty, I. Meex, R. Kuehl, S. Nijs, R. G. Richards, M. Raschke, O. Borens, S. L. Kates, C. Zalavras, P. V. Giannoudis and M. H. J. Verhofstad, Injury, 2018, 49, 497-504.

3 R. A. Henderson and M. S. Austin, J. Arthroplasty, 2017, 32, 2056-2059.

4 E. M. Hetrick and M. H. Schoenfisch, Chem. Soc. Rev., 2006, 35, 780-789.

5 S. Ferraris and S. Spriano, Mater. Sci. Eng., C, 2016, 61, 965978.

6 R. Kargupta, S. Bok, C. M. Darr, B. D. Crist, K. Gangopadhyay, S. Gangopadhyay and S. Sengupta, Wiley Interdiscip. Rev.: Nanomed. Nanobiotechnol., 2014, 6, 475-495.

7 J. A. Inzana, E. M. Schwarz, S. L. Kates and H. A. Awad, Biomaterials, 2016, 81, 58-71.

8 D. P. Lew and F. A. Waldvogel, Lancet, 2004, 364, 369-379.
9 A. J. Tande, E. O. Gomez-Urena, E. F. Berbari and D. R. Osmon, Infect. Dis. Clin. North Am., 2017, 31, 237-252. 10 R. O. Darouiche, N. Engl. J. Med., 2004, 350, 1422-1429.

11 T. N. Phan, T. Buckner, J. Sheng, J. D. Baldeck and R. E. Marquis, Oral Microbiol. Immunol., 2004, 19, 31-38.

12 H. Hu, W. Zhang, Y. Qiao, X. Jiang, X. Liu and C. Ding, Acta Biomater., 2012, 8, 904-915.

13 J. X. Wang, H. J. Zhou, G. Y. Guo, J. Q. Tan, Q. J. Wang, J. Tang, W. Liu, H. Shen, J. H. Li and X. L. Zhang, ACS Appl. Mater. Interfaces, 2017, 9, 33609-33623.

14 Y. Q. Liang, J. Xu, J. Chen, M. C. Qi, X. H. Xie and M. Hu, Mol. Med. Rep., 2015, 11, 4225-4231.

15 K. Yusa, O. Yamamoto, H. Takano, M. Fukuda and M. Iino, Sci. Rep., 2016, 6, 29462.

16 X. K. Shen, Y. Hu, G. Q. Xu, W. Z. Chen, K. Xu, Q. C. Ran, P. P. Ma, Y. R. Zhang, J. H. Li and K. Y. Cai, ACS Appl. Mater. Interfaces, 2014, 6, 16426-16440.

17 K. Li, J. M. Yu, Y. T. Xie, L. P. Huang, X. J. Ye and X. B. Zheng, J. Mater. Sci.: Mater. Med., 2011, 22, 2781-2789.

18 Y. H. Chen, G. W. Wu and J. L. He, Mater. Sci. Eng., C, 2015, 48, 41-47.

19 Y. Yu, G. Jin, Y. Xue, D. Wang, X. Liu and J. Sun, Acta Biomater., 2017, 49, 590-603.

20 J. He, W. Feng, B. H. Zhao, W. Zhang and Z. Lin, Int. J. Oral Maxillofac. Implants, 2018, 33, 298-310.

21 J. Bejarano, P. Caviedes and H. Palza, Biomed. Mater., 2015, 10, 025001.

22 M. Li, X. M. Liu, Z. Q. Xu, K. W. K. Yeung and S. L. Wu, ACS Appl. Mater. Interfaces, 2016, 8, 33972-33981.

23 N. Khoshnood, A. Zamanian and A. Massoudi, Mater. Sci. Eng., C, 2017, 77, 748-754.

24 H. Lee, J. Rho and P. B. Messersmith, Adv. Mater., 2009, 21, 431-434.

25 C. C. Gao, Y. Wang, F. X. Han, Z. Q. Yuan, Q. Li, C. Shi, W. W. Cao, P. H. Zhou, X. D. Xing and B. Li, J. Mater. Chem. B, 2017, 5, 9326-9336.

26 L. Wang, X. Yang, W. W. Cao, C. Shi, P. H. Zhou, Q. Li, F. X. Han, J. Y. Sun, X. D. Xing and B. Li, RSC Adv., 2017, 7, 51593-51604.

27 J. H. Kim, M. K. Joshi, J. Lee, C. H. Park and C. S. Kim, J. Colloid Interface Sci., 2018, 513, 566-574.

28 S. H. Ku, J. Ryu, S. K. Hong, H. Lee and C. B. Park, Biomaterials, 2010, 31, 2535-2541.

29 W. B. Tsai, W. T. Chen, H. W. Chien, W. H. Kuo and M. J. Wang, Acta Biomater., 2011, 7, 4187-4194.

30 S. Zhong, R. Luo, X. Wang, L. Tang, J. Wu, J. Wang, R. Huang, H. Sun and N. Huang, Colloids Surf., B, 2014, 116, 553-560. 31 S. Trouillet-Assant, M. Gallet, P. Nauroy, J. P. Rasigade, S. Flammier, P. Parroche, J. Marvel, T. Ferry, F. Vandenesch, P. Jurdic and F. Laurent, J. Infect. Dis., 2015, 211, 571-581.

32 H. Cheng, Y. Li, K. Huo, B. Gao and W. Xiong, J. Biomed. Mater. Res., Part A, 2014, 102, 3488-3499.

33 S. M. Kurtz, K. L. Ong, J. Schmier, F. Mowat, K. Saleh, E. Dybvik, J. Karrholm, G. Garellick, L. I. Havelin, O. Furnes, H. Malchau and E. Lau, J. Bone Jt. Surg., Am. Vol., 2007, 89a, 144-151. 
34 J. Raphel, M. Holodniy, S. B. Goodman and S. C. Heilshorn, Biomaterials, 2016, 84, 301-314.

35 K. J. Bozic, S. M. Kurtz, E. Lau, K. Ong, V. Chiu, T. P. Vail, H. E. Rubash and D. J. Berry, Clin. Orthop. Relat. Res., 2010, 468, 45-51.

36 K. J. Bozic, S. M. Kurtz, E. Lau, K. Ong, T. P. Vail and D. J. Berry, J. Bone Jt. Surg., Am. Vol., 2009, 91, 128-133.

37 E. M. Greenfield, Y. M. Bi, A. A. Ragab, V. M. Goldberg, J. L. Nalepka and J. M. Seabold, J. Biomed. Mater. Res., Part $B$, 2005, 72b, 179-185.

38 S. M. Kurtz, E. Lau, H. Watson, J. K. Schmier and J. Parvizi, J. Arthroplasty, 2012, 27, 61-65 e61.

39 R. J. Holleyman, P. Baker, A. Charlett, K. Gould and D. J. Deehan, Knee Surg. Sports Traumatol. Arthrosc., 2016, 24, 3080-3087.

40 J. Pajarinen, E. Jamsen, Y. T. Konttinen and S. B. Goodman, J. Long Term Eff. Med. Implants, 2014, 24, 283-296.

41 L. Pulido, E. Ghanem, A. Joshi, J. J. Purtill and J. Parvizi, Clin. Orthop. Relat. Res., 2008, 466, 1710-1715.

42 W. Zimmerli, A. Trampuz and P. E. Ochsner, N. Engl. J. Med., 2004, 351, 1645-1654.

43 B. H. Kapadia, R. A. Berg, J. A. Daley, J. Fritz, A. Bhave and M. A. Mont, Lancet, 2016, 387, 386-394.

44 M. Emmerson, New Horiz., 1998, 6, S3-S10.

45 K. G. Neoh, X. F. Hu, D. Zheng and E. T. Kang, Biomaterials, 2012, 33, 2813-2822.
46 C. Ning, X. Wang, L. Li, Y. Zhu, M. Li, P. Yu, L. Zhou, Z. Zhou, J. Chen, G. Tan, Y. Zhang, Y. Wang and C. Mao, Chem. Res. Toxicol., 2015, 28, 1815-1822.

47 K. M. Reddy, K. Feris, J. Bell, D. G. Wingett, C. Hanley and A. Punnoose, Appl. Phys. Lett., 2007, 90, 2139021-2139023.

48 G. Jin, H. Cao, Y. Qiao, F. Meng, H. Zhu and X. Liu, Colloids Surf., B, 2014, 117, 158-165.

49 P. Liu, Y. Zhao, Z. Yuan, H. Ding, Y. Hu, W. Yang and K. Cai, Mater. Sci. Eng., C, 2017, 75, 998-1005.

$50 \mathrm{~W}$. Watjen, H. Haase, M. Biagioli and D. Beyersmann, Environ. Health Perspect., 2002, 110(suppl. 5), 865-867.

51 A. G. Gristina, Science, 1987, 237, 1588-1595.

52 D. J. Schurman, B. L. Johnson Jr and H. C. Amstutz, J. Bone Jt. Surg., Am. Vol., 1975, 57, 40-49.

53 A. B. Lovati, M. Bottagisio, E. de Vecchi, E. Gallazzi and L. Drago, Adv. Exp. Med. Biol., 2017, 971, 29-50.

54 L. Gatin, A. Saleh-Mghir, P. Massin and A. C. Cremieux, Orthop. Traumatol. Surg. Res., 2015, 101, 851-855.

55 X. Lin, S. Yang, K. Lai, H. Yang, T. J. Webster and L. Yang, Nanomedicine, 2017, 13, 123-142.

56 K. Huo, X. Zhang, H. Wang, L. Zhao, X. Liu and P. K. Chu, Biomaterials, 2013, 34, 3467-3478.

57 Y. Qiao, W. Zhang, P. Tian, F. Meng, H. Zhu, X. Jiang, X. Liu and P. K. Chu, Biomaterials, 2014, 35, 6882-6897.

58 J. Josse, F. Velard and S. C. Gangloff, Front. Cell. Infect. Microbiol., 2015, 5, 85. 